\title{
Patterning of silicon surfaces with noncontact atomic force microscopy: Field-induced formation of nanometer-size water bridges
}

\author{
Ricardo García ${ }^{a)}$ and Montserrat Calleja \\ Instituto de Microelectrónica de Madrid, CSIC, Isaac Newton 8, 28760 Tres Cantos, Madrid, Spain \\ Heinrich Rohrer \\ Lab. Sistemas Pequeños y Nanotecnología, CSIC, Serrano 114, 28006 Madrid, Spain
}

(Received 3 March 1999; accepted for publication 27 April 1999)

\begin{abstract}
Nanometer-size water bridges have been used to confine the oxidation of silicon surfaces with a noncontact atomic force microscope. The formation of a water bridge between two surfaces separated by a gap of a few nanometers is driven by the application of an electrical field. Once a liquid bridge is formed, its length and neck diameter can be modified by changing the tip-sample separation. The liquid bridge provides the ionic species and the spatial confinement to pattern $\mathrm{Si}(100)$ surfaces in noncontact force microscopy. The method is applied to write arrays of several thousands dots with a periodicity of $40 \mathrm{~nm}$ and an average width of $10 \mathrm{~nm}$. (C) 1999 American Institute of Physics. [S0021-8979(99)06515-9]
\end{abstract}

\section{INTRODUCTION}

The emergence of the nanoscience and nanotechnology fields is partly based on the development of lithographic methods to pattern surfaces with structural features in the nanometer scale range. Scanning probe-based lithography has opened a variety of fascinating methods for the modification of metallic, ${ }^{1,2}$ semiconducting, ${ }^{3}$ biological, ${ }^{4}$ and organic surfaces. ${ }^{5,6}$ However, few of them seem suitable for large scale fabrication of nanostructures. The sample requirements (ultrahigh vacuum) in some cases or the extreme dependence on tip conditioning and shape in others have limited their application.

The direct oxidation of silicon, III-V semiconductors, titanium, and molybdenum surfaces by a conducting tip is arguably the most promising scanning probe microscopy (SPM) approach for the fabrication of nanoelectronic and nanomechanical devices. ${ }^{7-14}$ Recently this field has experienced a renovated interest. For one side, several results have contributed to an increased understanding of the oxidation mechanism in silicon surfaces ${ }^{15,16}$ and its kinetics. ${ }^{17-20} \mathrm{Si}$ multaneously, the reproducibility, aspect ratio and control of the oxide size have been improved by performing the oxidation with an oscillating tip while keeping the tip and the sample several nanometers apart ${ }^{20}$ and by modulating the tip-sample voltage. ${ }^{21}$

In this article we present a comprehensive study of the role of the water meniscus during the local oxidation of silicon surfaces by an atomic force microscope (AFM) probe placed a few nanometers above the sample [noncontact AFM (or SPM) oxidation]. Two major features distinguish this approach from static (contact) SPM oxidation. First, the cantilever is vibrated at its resonance frequency a few nanometers above the sample. Second, two voltages are used to locally oxidize the surface. An initial threshold voltage $V_{t h}$ is required to induce the formation of a water meniscus. The

\footnotetext{
${ }^{a)}$ Electronic mail: rgarcia@imm.cnm.csic.es
}

other voltage, $V_{o x}$ drives the oxyanions $\left(\mathrm{OH}^{-}, \mathrm{O}^{-}\right)$towards the $\mathrm{Si} / \mathrm{SiO}_{x}$ interface to generate the oxide.

Critical to the noncontact oxidation is the formation of a nanometer-size liquid bridge between tip and sample. The liquid bridge provides the oxygen species, as well as, the pathway for the electrical field to induce the oxidation. Evidence of the bridge formation is obtained from changes in the cantilever dynamics and by measuring the capillary force acting on the cantilever. The effective confinement of the oxidation within the limits of the bridge allows the control of the lateral dimension of the oxide marks (dots). We show this by modulating the liquid neck with the tip-sample separation.

Earlier contact SPM oxidations have already pointed out that the direct oxidation requires the presence of a water film adsorbed onto the surface. ${ }^{17,22}$ The contact between the tip and the sample in humid air drives the spontaneous formation of a water meniscus whose diameter may reach several hundreds of nanometers. ${ }^{23}$ As a consequence, the oxidation involved is extended on a large effective area. This effect usually limits the spatial resolution as well as the homogeneity of the lithographic process. Those limitations may be avoided by performing the oxidation with the tip and the sample separated by a gap of several nanometers. If the gap between the surfaces is larger than twice the Kelvin radius, an external force is needed to drive the formation of a water meniscus. An electrical field is applied to form a water bridge connecting the tip and the sample. We found that for a given relative humidity and tip-sample separation, a voltage above a certain threshold must be applied to form the bridge.

We show that the field-induced bridges may have characteristic dimensions, length, and neck diameter, in the nanometer range. We also describe the use of those bridges to produce the electrochemical oxidation of silicon surfaces. The potential technological applications are illustrated by generating 4864 dots with a $40 \mathrm{~nm}$ periodicity and an esti- 
mated width of $10 \mathrm{~nm}$. The estimated diameter of the dots emphasizes the nanometer character of the water bridges.

In previous contributions ${ }^{16,20}$ we have demonstrated that the oxidation kinetics of silicon surfaces in noncontact follows the same behavior than in contact SPM. As a consequence the results obtained by Dagata et al. ${ }^{15}$ about the oxidation mechanism should also apply here. Those aspects will not be discussed in this article.

The article is organized as follows. In Sec. II the experimental details are described. The changes in the cantilever dynamics due to the water bridge formation and the physical origin of the threshold voltage are analyzed in Sec. III. Section IV emphasizes the relationship between the liquid bridge and lateral extent of the oxide. An illustration of the noncontact SPM oxidation for patterning surfaces is shown in Sec. V. Finally, in Sec. VI the main conclusions of this work are summarized.

\section{EXPERIMENTS}

The experiments were performed with an atomic force microscope (AFM) operated in a noncontact mode (Nanoscope III, Digital Instruments) with additional circuits to perform oxidation. Silicon cantilevers were metallized with a layer of $30 \mathrm{~nm}$ of Ti. The average force constant $\left(k_{c}\right)$ and resonance frequency $\left(f_{0}\right)$ were about $34 \mathrm{~N} / \mathrm{m}$ and $330 \mathrm{kHz}$, respectively. The samples were $p$-type $\mathrm{Si}(100)$ with a resistivity of $14 \Omega \mathrm{cm}$. To eliminate organic contaminants, the samples were sonicated for $60 \mathrm{~s}$ first in acetone and then in ethanol. For environmental control, the microscope was placed into a closed box with inlets for dry and $\mathrm{H}_{2} \mathrm{O}$ saturated nitrogen. Due to exposure to air, the silicon surface has a native oxide layer of about $2 \mathrm{~nm}$.

The cantilever is oscillated at its free resonance frequency. The damping regime of the force microscope (noncontact and attractive) is controlled by recording the phase shift dependence on the tip-sample separation. The position of the sample with respect to the tip is derived from the measurement of amplitude and cantilever's deflection curves. To record the time evolution of the amplitude, the signal from the photodiode has been acquired with an oscilloscope. In Fig. 1 the criterion used to measure the tip-sample average separation is schematized. This criterion is in full agreement with computer simulations. ${ }^{24}$

To estimate the tip radius of curvature, a test sample of InSb quantum dots on InP (Ref. 25) is imaged. Transmission electron microscopy images show the dots as truncated pyramids with well defined facets. For a given tip, the comparison performed between the apparent AFM image and the dot's geometry allows to estimate the tip radius.

The protocol to form liquid bridges on $\mathrm{Si}(100)$ surfaces requires several steps that are schematized in Fig. 2. First, a few monolayers of water are adsorbed onto the surface [Fig. 2(a)]. Hydrophilic surfaces show the spontaneous adsorption of water from the vapor phase if they are kept at a relative humidity (RH) above 30\%-40\% (Refs. 26-29). Next, a force microscope cantilever/tip is oscillated a few nanometers above the sample surface, then a first voltage pulse of several $\mathrm{ms}$ is applied. The electrical field (pulse) polarizes

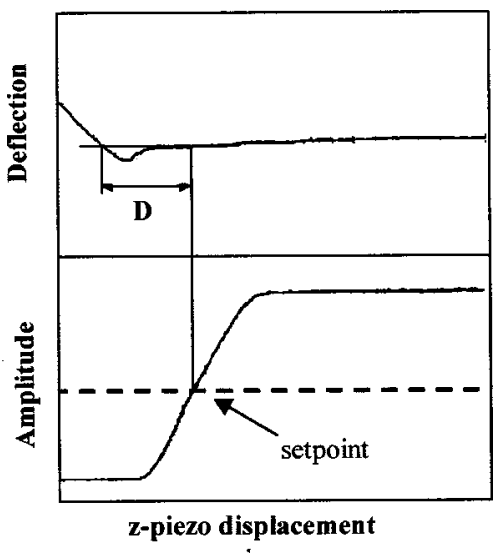

FIG. 1. Determination of the tip and sample separation from deflection and amplitude vs $z$-piezo displacement. The origin (zero tip-sample distance) is the $z$-piezo position where repulsive and attractive forces are equal. The intersection of the set point amplitude at the $z$-piezo displacement set gives the actual tip position. The difference between this value and the $z$-displacement at zero deflection is the tip-sample $D$ distance.

the water layer in the proximity of the tip's apex. If the voltage is above a certain threshold value $\left(V_{t h}\right)$ a water bridge between tip and sample is formed [Fig. 2(b)]. Once the bridge is formed, another voltage pulse $V_{o x}$ (sample positive) is applied to grow the oxide [Fig. 2(c)]. In some cases $V_{t h}$ has also been used as $V_{o x}$.

\section{PHYSICAL ORIGIN OF THE THRESHOLD VOLTAGE}

\section{A. Liquid bridge formation}

In a previous contribution, we have observed that the oxidation in noncontact force microscopy requires the application of a threshold voltage. ${ }^{20}$ Tip-sample voltages smaller than $V_{t h}$ do not produce any modification of the surface

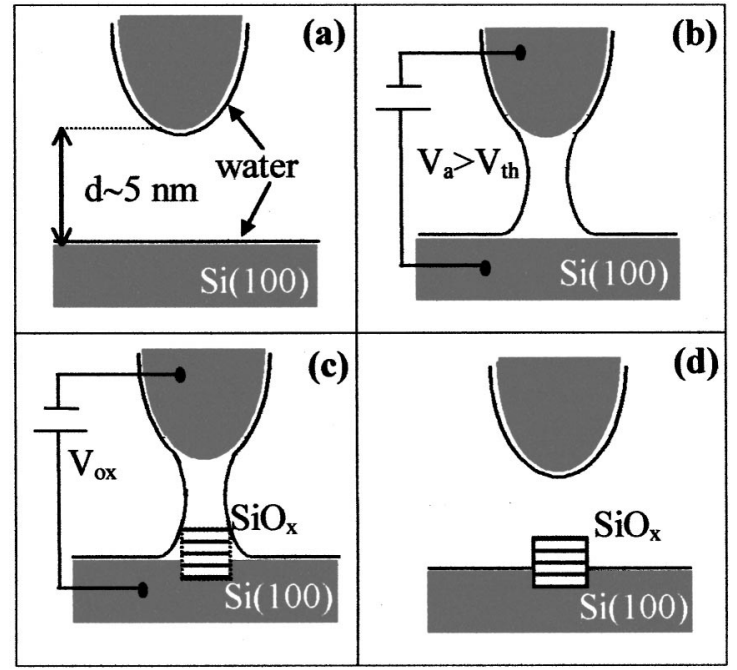

FIG. 2. Steps on the oxidation of $\mathrm{Si}(100)$ in dynamic force microscopy. (a) An oscillating tip is approached towards the surface up to a minimum equilibrium distance of about $5 \mathrm{~nm}$. (b) An external voltage polarizes the water monolayers adsorbed onto the surface. If $V_{a}$ is above $V_{t h}$ a bridge is formed. (c) A voltage pulse $V_{o x}$ is applied to induce the oxidation of the silicon. (d) The tip is withdrawn and the dot is imaged. 


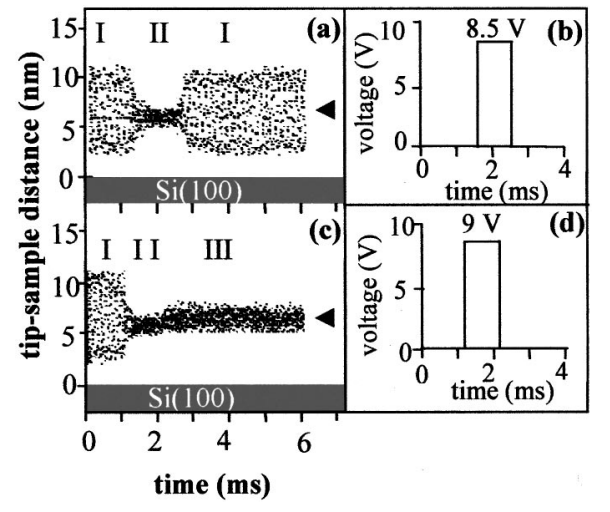

FIG. 3. Tip's oscillation amplitude and applied voltage vs time for two $1 \mathrm{~ms}$ pulses. (a) $V_{a}=8.5 \mathrm{~V}$; (c) $V_{a}=9 \mathrm{~V}$. During the pulse, a reduction of the amplitude and a shift of the tip's equilibrium position towards the surface are observed in both cases (II). However, in (c) some amplitude damping and tip's shift remain after the pulse (III). The position of the $\mathrm{Si}(100)$ surface has been plotted, so the instantaneous tip-sample separation can be deduced. The arrows indicate the initial tip's average position.

while the application of voltages above $V_{t h}$ induce the local oxidation of the surface. In this section, we study the cantilever dynamics under the application of a voltage pulse to determine the physical origin of $V_{t h}$.

Figure 3 shows the tip's oscillation before, during, and after the application of two $1 \mathrm{~ms}$ pulses of 8.5 and $9 \mathrm{~V}$, respectively. During the first pulse $\left(V_{a}=8.5 \mathrm{~V}\right)$, the cantilever's equilibrium position is deflected $0.6 \pm 0.1 \mathrm{~nm}$ towards the surface and the amplitude is reduced by a factor 5 (II). After the pulse, the cantilever recovers its initial amplitude and equilibrium position (I), Fig. 3(a). The voltage pulse is plotted in Fig. 3(b). The electrostatic force is responsible for the deflection and the amplitude reduction. A few seconds later a second pulse at $V_{a}=9 \mathrm{~V}$ is applied [Fig. 3(d)]. The cantilever's deflection is about $0.8 \pm 0.1 \mathrm{~nm}$. However, after turning off the voltage, the cantilever does not recover its original equilibrium position. A deflection of about 0.3 $\pm 0.1 \mathrm{~nm}$ is still observed. Additionally, the amplitude remains damped for several minutes (III) [Fig. 3(c)]. After repeating the experiment for other voltages, the conclusions have been the same. Voltages below $8.8 \pm 0.2$ are described by an oscillation similar to the one depicted in Fig. 3(a) while voltages above $8.8 \pm 0.2$ belong to the class depicted in Fig. 3(c). To illustrate the noncontact character of the process the position of the surface with respect to the tip is also plotted. The closest tip-sample separation is above $2 \mathrm{~nm}$ [ $\mathrm{I}$ in Fig. 3(a)].

The state III reveals the presence of an attractive force of $-10 \mathrm{nN}(F=-k \times \Delta z)$. We attribute this force to the formation of a water bridge. Additional evidence of the presence of a meniscus may be found by studying the changes in the cantilever dynamics. The force gradient associated with the capillary force should shift the cantilever resonance curve to lower frequencies. The meniscus viscosity would also imply a reduction of the quality factor $Q$ of the cantilever. The quality factor is estimated by $Q=f_{0} / \Delta f$ with $\Delta f$ as the full width at $1 / \sqrt{ } 2$ th maximum of the resonance curve. We have recorded the amplitude dependence upon driving frequency for the free cantilever and for the same cantilever when it is

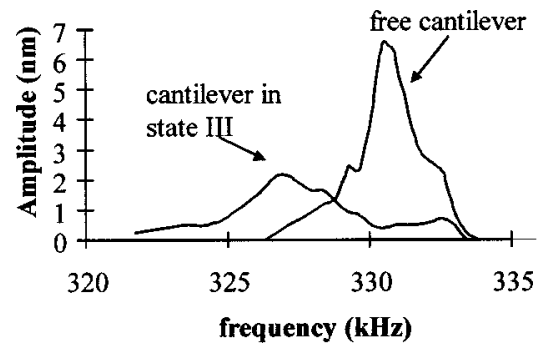

FIG. 4. Amplitude vs frequency for the free cantilever and for the same cantilever (tip) after the formation of state III. $\mathrm{RH}=55 \%$.

placed in state III (Fig. 4). The curve for the free cantilever has its peak at $330.9 \mathrm{kHz}$ and a $Q$ of about 399. After a voltage pulse of $9 \mathrm{~V}$ for $1 \mathrm{~ms}$, the resonance is shifted to $326.9 \mathrm{kHz}$ and $Q$ decreases to 100 (Fig. 4). The frequency shift implies a positive force gradient of about $0.9 \mathrm{~N} / \mathrm{m}$ $\left(\delta F / \delta z \cong-2 k_{c} \Delta f / f_{0}\right)$.

The capillary force for a SPM tip of arbitrary shape and a flat sample can only be determined by the numerical solution of the Young-Laplace differential equation. However, for a spherical tip the capillary force can be estimated ${ }^{30,31}$ by

$$
F_{C}=\pi \gamma_{L} \kappa R^{2} \sin ^{2} \phi
$$

and the force gradient by,

$$
\frac{\partial F_{C}}{\partial D} \approx 2 \pi \gamma_{L} \kappa R^{2} \sin \phi \cos \phi \frac{d \phi}{d D},
$$

where $R$ is the apex radius, $\gamma_{L}$ is the water surface energy, $\phi$ is the filling angle, $D$ is the tip-sample distance (see scheme in Fig. 5), and $\kappa$ is the mean meniscus curvature given by,

$$
\kappa=-\frac{\cos \phi+1}{R(1-\cos \phi)+D}+\frac{1}{R} .
$$

To deduce above expressions, we have made several simplifying assumptions. First, the contact angles between the liquid and the tip $\theta_{1}$, and the liquid and the sample $\theta_{2}$ are assumed to be equal to zero. This implies that the liquid wets the surfaces. Second, the contribution to the capillary force due to the vertical component of the surface tension force acting along the perimeter of the sphere has been neglected. Third, we have also neglected the buoyancy force due to

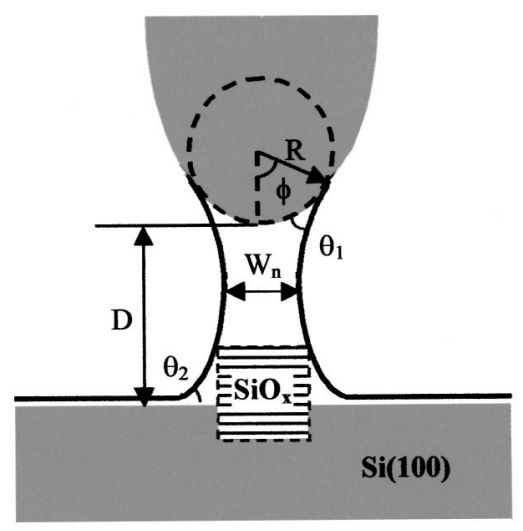

FIG. 5. Model of the tip-liquid bridge-surface interface used here. $W_{n}$ represents the liquid bridge neck diameter. 


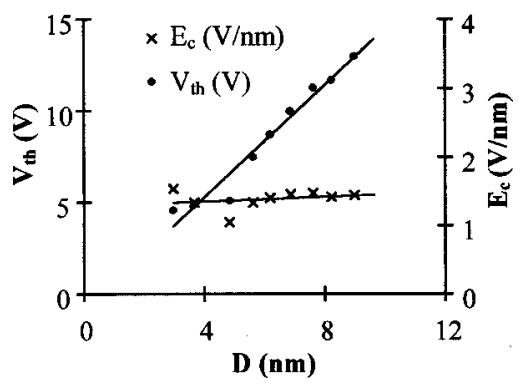

FIG. 6. Threshold voltage and electrical field dependence of the bridge formation on the average tip-sample distance. $\mathrm{RH}=31 \%$. The solid lines are linear fittings.

gravity. Previous studies determined that gravitational effects are negligible when the amount of trapped liquid is small, ${ }^{30}$ which fully applies here. This implies that the meniscus everywhere has the same mean curvature. Here, we have modeled the meridional profile of the meniscus by an arc of circle. In the limit of small $\phi$, Eq. (1) coincides with the expression deduced in Ref. 32.

From Eq. (1) a force of $-7.7 \mathrm{nN}$ is obtained $(R$ $=30 \mathrm{~nm}, D=7 \mathrm{~nm}$, and $\gamma_{L}=73 \mathrm{~mJ} / \mathrm{m}^{2}$ ). This value is comparable with the experimental force of $\sim-10 \mathrm{nN}$ deduced from Fig. 3(c). The force gradient derived from Eq. (2) is 1 $\mathrm{N} / \mathrm{m}$, i.e., in excellent agreement with the experimental result of $0.9 \mathrm{~N} / \mathrm{m}$. From this comparison we conclude that $V_{t h}$ is related to the formation of a liquid bridge between the tip and the sample. In addition, Fig. 3(c) is also used to establish a practical criterion to determine the formation of a liquid bridge. Furthermore, similarity between $\operatorname{contact}^{17,18}$ and noncontact ${ }^{20}$ oxidation kinetics underlines the presence of a water meniscus in both cases.

\section{B. Threshold voltage dependence on tip-sample separation}

We have also performed experiments to determine whether the process of a liquid bridge formation is a voltage or field dependent process. First, we have observed that the same experimental conditions (tip radius, relative humidity, and sample separation) of the absolute value of $V_{t h}$ was independent of the polarity.

Second, we have measured the threshold voltage as a function of the tip-sample average distance $D$. We have calculated the ratio $V_{t h} / D$, i.e., the average electrical field for the formation of the bridge as a function of $D$ (Fig. 6). $V_{t h}$ shows a linear increase with $D$ from $5 \mathrm{~V}$ for $D=3 \mathrm{~nm}$ to 14 $\mathrm{V}$ for $D=10 \mathrm{~nm}$. However, the ratio $V_{t h} / D$ remains roughly constant $\sim 1.3 \mathrm{~V} / \mathrm{nm}$. The curvature radius of the tips used in these experiments are about $15-50 \mathrm{~nm}$, i.e., 2 to 5 times larger than $D$ values, then the electrical field on the surface and underneath the tip can be approximated by $E=V / D$. The above result emphasizes the existence of a critical field given by $E_{C}=V_{t h} / D$ as the main requirement for the formation of a liquid bridge. This field would be the minimum electrical field needed to polarize and pull the water molecules adsorbed on the surface towards the tip. The thickness of the water layer adsorbed on the surface and the meniscus curva-
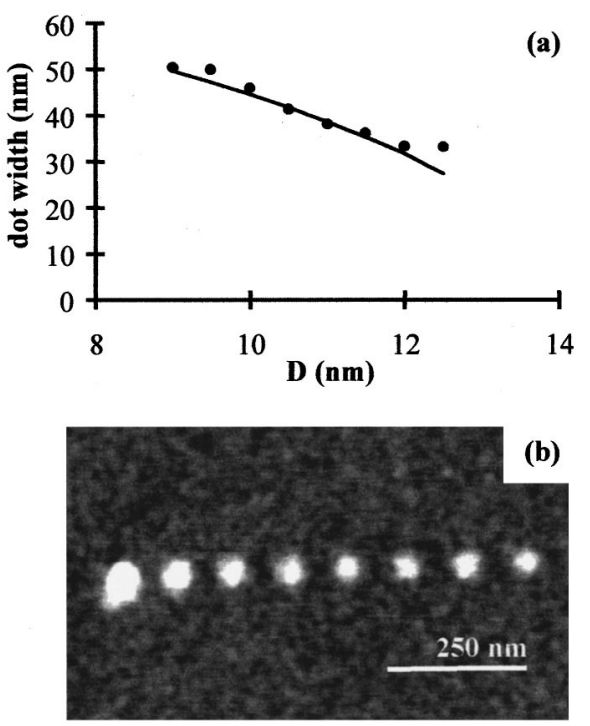

FIG. 7. Dot width dependence on the tip-sample average distance. First the bridge (state III) is formed by a $0.5 \mathrm{~ms}$ pulse of $18 \mathrm{~V}$, then tip and sample are separated. Finally, the dots are grown by the application of a pulse of 8 $\mathrm{V}$ for $0.3 \mathrm{~s}$. The solid line represents the variation of the neck diameter with the distance for the model shown in the inset. $\mathrm{RH}=50 \%$.

ture depend on the relative humidity $(\mathrm{RH})$. Accordingly $E_{C}$ should decrease with RH. This effect has been confirmed experimentally.

\section{EFFECTIVE CONFINEMENT OF THE LOCAL OXIDATION WITHIN THE LIMITS OF THE LIQUID NECK}

The local oxidation by SPM techniques requires the existence of a water meniscus connecting tip and sample. The water meniscus provides the oxyanions $\left(\mathrm{OH}^{-}, \mathrm{O}^{-}\right)$for the oxidation to proceed. In contact SPM oxidation, the thermodynamics favors the spontaneous formation of a meniscus. In noncontact AFM an external electrical force drives its formation. Additionally, noncontact AFM offers a procedure to change the length and the neck diameter of the bridge by modifying the tip-sample separation. Temporal stability and changeable geometry are key factors to use the bridges to confine a chemical reaction, such as the oxidation of $\mathrm{Si}(100)$ surfaces. In this section, we study the relationship between the bridge neck size and the lateral extent of the oxide.

The experiment requires the application of two different voltage pulses, one to form the liquid bridge and the other to drive the oxyanions to the $\mathrm{Si} / \mathrm{SiO}_{2}$ interface and to grow the oxide. First, a $0.5 \mathrm{~ms}$ pulse of $18 \mathrm{~V}$ is applied to build the bridge. This pulse was well above $V_{t h}=11 \mathrm{~V}$. Then, tip and sample are separated, i.e., the bridge is stretched. Finally, to grow a dot an oxidation pulse of $V_{o x}=8 \mathrm{~V}$ and, $0.3 \mathrm{~s}$ is applied. The experimental data (circles) show that the width of the oxide decreases by increasing the tip-sample separation (Fig. 7). The width is reduced from 50 to $30 \mathrm{~nm}$ by increasing the separation from 8.5 to $12 \mathrm{~nm}$.

Next, we have calculated the dependence of the neck diameter on the separation for a meniscus characterized by Eq. (3). The results are plotted by a continuous line in Fig. 7. The almost one to one correlation obtained between the neck and the width of the oxide is attributed to two factors. First, 


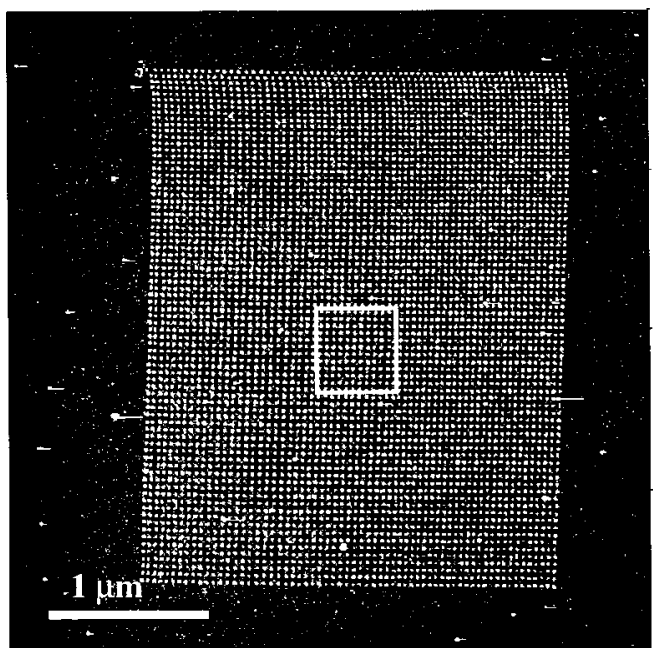

FIG. 8. AFM image of an array of 4864 dots. Before growing a dot a new bridge is formed. The dots are $40 \mathrm{~nm}$ apart and with an average width of 10 $\mathrm{nm}$. The same tip was used to grow the dots and to image them afterwards.

the bridge provides the pathway for the oxygen species to oxide the silicon. More importantly, the difference between the electrical permittivity inside and outside the liquid bridge, 81 and 1, respectively, acts as an effective lens, focusing the fieldlines within the neck limits. The result is that the ions involved in the oxidation are effectively confined within a cylinder of diameter the neck of the bridge. In addition, the above findings emphasize the true threedimensional nanometer size of the liquid bridges.

\section{PATTERNING OF THE SILICON SURFACES}

The results described in previous sections are some of the main elements needed to develop a method to pattern silicon surfaces with a conducting probe placed several nanometers above the surface. The reproducibility of the fieldinduced formation of liquid bridges is shown by writing 4864 dots on $\mathrm{Si}(100)$ with a periodicity of $40 \mathrm{~nm}$ (Fig. 8). In terms of a storage memory device, the dot density is approximately $1 \mathrm{~Tb} / \mathrm{in}^{2}$. Each dot has been generated by the application of a single voltage pulse of $V_{o x}=17 \mathrm{~V}$ for $0.3 \mathrm{~ms}$. In this case, $V_{o x}$ was higher than the threshold $V_{t h}=9.5 \mathrm{~V}$. In other words, the same pulse has been used to form the bridge and to grow the oxide. Between dots the feedback is restored and the tip-sample separation adjusted.

A zoom in the central region of Fig. 8 illustrates the potential for patterning motives of $10 \mathrm{~nm}$ (Fig. 9). The apparent AFM cross-sections of two dots are shown (solid lines). The dashed line and shaded region are the upper and lower limits of the reconstructed image [Fig. 9(b)]. The region in between is the unreconstructable region. ${ }^{33}$ The reconstructed width of the dots lie between 5 and $16 \mathrm{~nm}$ for the first, and 4 and $15 \mathrm{~nm}$ for the second. From this reconstruction we infer that the dot's width is in $4-16 \mathrm{~nm}$ range, with a mean value of $10 \mathrm{~nm}$. The reconstruction has been obtained with a parabolic tip of apex radius $51 \mathrm{~nm} . R=51 \mathrm{~nm}$ was the estimated radius of the tip used to generate this image.

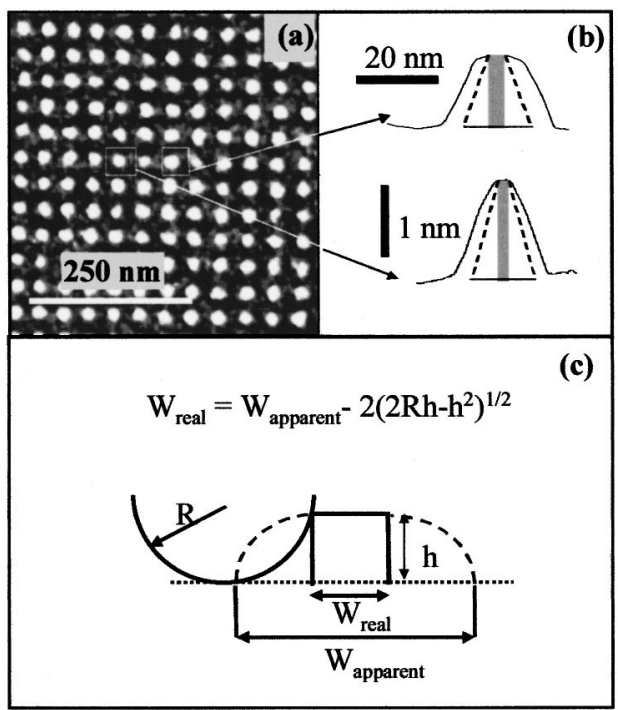

FIG. 9. (a) High resolution image of the region marked in Fig. 8. (b) Minimum (shaded) and maximum (dashed line) cross-sections of the dots encircled in (a) after image reconstruction. To reconstruct the images trapezoidal and rectangular geometries have been assumed. The solid line is the apparent AFM image. (c) Scheme of an image reconstruction for a rectangular protrusion and a spherical tip. Tip's curvature radius $\sim 51 \mathrm{~nm}$. $V_{o x}$ $=17 \mathrm{~V}$ and $0.3 \mathrm{~ms}$, oscillation amplitude $5.3 \mathrm{~nm}, \mathrm{RH}=30 \%$.

\section{SUMMARY}

In conclusion, we have shown a method to pattern silicon surfaces by noncontact force microscopy. A key feature of the method is the field-induced formation of a water bridge between two surfaces separated by a gap of a few nanometers. The existence of a threshold voltage is directly connected to the critical electrical field needed to polarize and pull the water layer towards the tip. Evidence of the bridge formation is provided by analyzing the changes in the oscillation amplitude and in the resonance curves due to the attractive meniscus force.

The details of the mechanism involved in the direct oxidation of silicon surfaces in the presence of water and an electrical field are complex, nevertheless, the oxidation follows the overall chemical reaction $\mathrm{Si}+4 h^{+}+2 \mathrm{OH}^{-}$ $\rightarrow \mathrm{SiO}_{2}+2 \mathrm{H}^{+}$. Due to the electrochemical nature of the oxidation a compromise between the dimensions of the water bridge and the availability of ionic species for the oxidation to proceed must be reached. An estimation shows that a cylindrical bridge $10 \mathrm{~nm}$ long and a neck diameter of $4 \mathrm{~nm}$ contains enough oxygen species to form a $4 \mathrm{~nm}$ dot.

As a method to pattern surfaces noncontact (nc)-AFM offers two main advantages with respect to contact SPM oxidation. The tip lifetime is greatly enhanced due to the absence of contact forces. This increases the reproducibility to pattern surfaces at large scale. The lateral confinement of the oxidation reaction within the limits of the neck, whose size can be modified, allows to control the lateral width of the oxide marks. The potential of the technique to make structures for use in devices or fundamental studies of nanometersize systems has been illustrated by the robustness of the process, its simplicity, size control, and the potential for 10 $\mathrm{nm}$ patterning. The method could also be applied to form 
liquid bridges of other polar solvents, so many other chemical reactions could be confined in a three-dimensional nanometer-size region.

\section{ACKNOWLEDGMENTS}

We acknowledge fruitful discussions with F. Briones, N. García, F. Pérez-Murano, and J. J. Sáenz. The work was supported by the Commission of the European Communities (Project 22955, LASMEDS), Comunidad Autónoma de Madrid and the DGICYT of Spain (PB-94-0016).

${ }^{1}$ R. M. Nyffenegger and R. M. Penner, Chem. Rev. 97, 1195 (1997) and references therein.

${ }^{2}$ H. J. Mamim, P. H. Guethner, and D. Rugar, Phys. Rev. Lett. 65, 2118 (1990).

${ }^{3}$ J. W. Lyding, T. C. Shen, J. S. Hubacek, J. R. Tucker, and C. Abeln, Appl. Phys. Lett. 64, 2010 (1994).

${ }^{4}$ R. García, Appl. Phys. Lett. 64, 1162 (1994).

${ }^{5}$ C. Marrian, E. S. Snow, Microelectron. Eng. 32, 173 (1996) and references therein.

${ }^{6}$ R. Piner, J. Zhu, F. Xu, S. Hong, and C. Mirkin, Science 283, 661 (1998).

${ }^{7}$ J. A. Dagata, J. Schneir, H. H. Harary, C. J. Evans, M. T. Postek, and J. Bennet, Appl. Phys. Lett. 56, 2001 (1990).

${ }^{8}$ H. C. Day and D. R. Alle, Appl. Phys. Lett. 62, 2691 (1993).

${ }^{9}$ F. Pérez-Murano, G. Abadal, N. Barniol, X. Aymerich, J. Servat, P. Gorostiza, and F. Sanz, J. Appl. Phys. 78, 6797 (1995).

${ }^{10}$ S. C. Minne, H. T. Soh, Ph. Flueckiger, and C. F. Quate, Appl. Phys. Lett. 66, 703 (1995)

${ }^{11}$ E. S. Snow, D. Park, and P. M. Campbell, Appl. Phys. Lett. 69, 269 (1996).

${ }^{12}$ K. Matsumoto, M. Ishii, and K. Segawa, J. Vac. Sci. Technol. B 14, 1331 (1996); K. Matsumoto, Proc. IEEE 85, 612 (1997).

${ }^{13}$ R. Held, T. Vancura, T. Heinzel, K. Ensslin, M. Holland, and W. Webscheider, Appl. Phys. Lett. 73, 262 (1998).
${ }^{14}$ A. Boisen, K. Birkelund, O. Hansen, and F. Grey, J. Vac. Sci. Technol. B 9, 2977 (1998).

${ }^{15}$ J. A. Dagata, T. Inoue, J. Itoh, K. Matsumoto, and H. Yokoyama, J. Appl. Phys. 84, 6891 (1998).

${ }^{16}$ M. Calleja, J. Anguita, R. García, K. Birkelund, F. Pérez-Murano, and J. Dagata, Nanotechnology 1, 10 (1999).

${ }^{17}$ P. Avouris, T. Hertel, and R. Martel, Appl. Phys. Lett. 71, 285 (1997).

${ }^{18}$ D. Stievenard, P. A. Fontaine, and E. Dubois, Appl. Phys. Lett. 70, 3272 (1997).

${ }^{19}$ F. Marchi, V. Bouchiat, H. Dallaporta, V. Safarov, D. Tonneau, and D. Poppelt, J. Vac. Sci. Technol. B 16, 1 (1998).

${ }^{20}$ R. García, M. Calleja, and F. Pérez-Murano, Appl. Phys. Lett. 72, 2295 (1998).

${ }^{21}$ F. Pérez-Murano, K. Birkelund, G. Abadal, K. Morimoto, and J. Dagata (unpublished).

${ }^{22}$ H. Sugimura and N. Nakagiri, Jpn. J. Appl. Phys., Part 1 34, 3406 (1995).

${ }^{23}$ J. Israelachvili, Intermolecular and Surface Forces (Academic, London, 1992).

${ }^{24}$ R. García and A. San Paulo, Phys. Rev. B (in press).

${ }^{25}$ J. Tamayo, R. García, T. Utzmeier, and F. Briones, Phys. Rev. B 55, R13436 (1997).

${ }^{26}$ D. Beaglehole, E. Z. Radlinska, B. W. Ninham, and H. K. Christenson, Phys. Rev. Lett. 66, 2084 (1991).

${ }^{27}$ L. Xu, A. Lio, J. Hu, D. Ogletree, and M. Salmeron, J. Phys. Chem. 102, 540 (1998).

${ }^{28}$ J. Colchero, A. Storch, M. Luna, J. Gómez-Herrero, and A. M. Baró, Langmuir 14, 2230 (1998).

${ }^{29}$ R. D. Piner and C. A. Mirkin, Langmuir 13, 6864 (1997).

${ }^{30}$ F. M. Orr, L. E. Scriven, and A. P. Rivas, J. Fluid Mech. 67, 723 (1975).

${ }^{31}$ E. Bayramli, A. Abou-Obeid, and T. G. M. Van de Ven, J. Colloid Interface Sci. 116, 490 (1987).

${ }^{32}$ C. M. Mate, M. R. Lorenz, and V. J. Novotny, J. Chem. Phys. 90, 7550 (1989).

${ }^{33}$ D. J. Keller and F. S. Franke, Surf. Sci. 294, 409 (1993). 